\title{
Tailoring Empirical Antimicrobial Therapy in Subjects With Ventilator-Associated Pneumonia With a 10-Hour E-Test Approach
}

\author{
Alexandre Boyer, Julien Goret, Benjamin Clouzeau, Antoine Romen, Renaud Prevel, \\ Edouard Lhomme, Frédéric Vargas, Gilles Hilbert, Cecile Bébéar, Didier Gruson, and \\ Fatima M'Zali
}

BACKGROUND: In a previous study of subjects suspected of having ventilator-associated pneumonia, a rapid susceptibility testing approach by using ETEST (BioMérieux) strips directly applied to bronchoalveolar lavage samples provided valuable information at hour 24 . The primary objective of this study was to assess a new direct specimen testing by using an even more-rapid E-test approach (at hour 10), which could promote an early de-escalation of the antimicrobial therapy. METHODS: Twenty-eight subjects with ventilator-associated pneumonia admitted to a medical ICU were prospectively included. In parallel with standard routine methods, E-test strips were directly applied onto agar plates seeded with bronchoalveolar lavage samples and were analyzed after $10 \mathrm{~h}$ of incubation. E-test results were used to identify potential drug choices by simulating clinical decision making if the microscopy results had been available at the point of care. These choices were analyzed for concordance with the narrowest adequate antimicrobial therapy according to the Minimum Inhibitory Concentrations (MICs) provided by the reference method (ie, the laboratory routine diagnostic). RESULTS: At hour 10, direct specimen testing was readable in 18 of 28 bronchoalveolar lavage samples $(64 \%)$. Total agreement between the 10 -h direct specimen testing approach and the laboratory routine diagnostic approach was $90 \%$, with a sensitivity of $83 \%$ and a specificity of $95 \%$, with $8 \%$ major errors and $3 \%$ very major errors. The concordance between the 2 tests was very good (kappa $=0.79)$. If the 10 -h E-test results were taken into account, then an early de-escalation strategy would have been possible in 10 of 18 cases $(55 \%)$ at hour 10 . CONCLUSIONS: This rapid susceptibility testing approach provided early $(10 \mathrm{~h})$ and valuable information that could lead to an early adjustment of empirical antimicrobial treatment in a ventilator-associated pneumonia setting. (ClinicalTrials.gov registration NCT01266863.) Key words: bronchoalveolar lavage; intensive care unit; direct antimicrobial testing; nosocomial pneumonia; ventilator-acquired pneumonia; rapid diagnostic. [Respir Care 2019;64(3):307-312. () 2019 Daedalus Enterprises]

\section{Introduction}

Patients who develop ventilator-associated pneumonia (VAP) in the ICU need adequate empirical antimicrobial

\footnotetext{
Drs Boyer, Clouzeau, Romen, Prevel, Vargas, Hilbert, and Gruson are affiliated with CHU de Bordeaux, Service de Réanimation Médecine Intensive, Bordeaux, France. Drs Boyer, Prevel, Gruson, and M'Zali are affiliated with Univ. de Bordeaux, UMR 5234 CNRS 'Microbiologie Cellulaire et Moléculaire et Pathogénicité', Bordeaux, France. Drs Goret and Bébéar are affiliated with CHU de Bordeaux, Service de Bactériologie, Bordeaux, France. Dr Lhomme is affiliated with CHU Bordeaux, Pôle de santé publique, CIC1401-EC, Bordeaux, France.
}

therapy to decrease VAP-related hospital mortality. ${ }^{1-3}$ Moreover, the rapid de-escalation of empirical broad-spec-

\footnotetext{
Part of this study was presented at the 54th European Congress of Clinical Microbiology and Infectious Diseases, held April 13, 2014, in Barcelona, Spain.

The authors have disclosed no conflicts of interest.

Correspondence: Alexandre Boyer MD PhD, Service de Réanimation Médecine Intensive, CHU Bordeaux, F-33000, Bordeaux, France. E-mail: alexandre.boyer@chu-bordeaux.fr.
}

DOI: $10.4187 /$ respcare.06255 
trum antimicrobial therapy is an important goal in the prevention of multidrug-resistant bacteria selection, together with avoiding the risk of overtreatment and toxicity. ${ }^{4}$ Direct specimen testing allows a rapid determination of bacterial susceptibility to antibiotics. Direct specimen testing by using ETEST (BioMérieux) strips on agar plates seeded with endotracheal aspirates from subjects with suspected VAP has been assessed ${ }^{5,6}$ and proved to give accurate results up to $3 \mathrm{~d}$ faster than standard techniques. ${ }^{6}$

We applied this promising direct specimen testing method to bronchoalveolar lavage (BAL) samples of subjects suspected of having VAP. This rapid susceptibility testing approach provided valuable information at $24 \mathrm{~h}^{7}$ We then improved the direct specimen testing method to allow faster results after only $10 \mathrm{~h}$ of incubation of the clinical samples. This could be helpful to physicians, who are known to rely on microbiology results for their decision to de-escalate because this could prompt an earlier de-escalation of care. The antibiotics exposition window sufficient to induce resistance is not known, but there should be an advantage to reducing patient exposure to broad empirical antibiotic therapy by several hours. ${ }^{8}$ The objective of the present study was to assess whether this new approach could provide accurate microbiology results to promote earlier de-escalation of antimicrobial therapy.

\section{Methods}

\section{Study Design and Subjects}

This protocol was conducted according to the same design used in our previous study. ${ }^{7}$ Subjects were recruited on admission to the medical ICU of Bordeaux University Hospital (13 beds) in southwest France, when the following criteria were met: on ventilation; $\geq 18$ y old; presented with standard criteria for suspicion of VAP (Clinical Pulmonary Infection Score $\geq 6$ ), and BAL and E-test approach was feasible. Patients who were undergoing antibiotic treatment for $>8 \mathrm{~h}$, had a contraindication to bronchoscopy, were pregnant, or who were expected to die within $48 \mathrm{~h}$ were excluded. BALs were performed according to a previously described procedure. ${ }^{9}$ The ethics committee of the Société de Réanimation de Langue Française approved the study protocol (07-204). Informed consent was waived because no supplementary procedure or biologic sampling were performed.

\section{Susceptibility Testing of BAL Samples}

The BAL fluid from each subject was divided into 2 samples. The first sample was sent to the routine diagnostic laboratory. Antibiotic susceptibilities results were classified as susceptible, intermediate, and resistant by the Phoenix automated microbiology system (BD Diagnostics, le Pont de

\section{QUICK LOOK}

\section{Current knowledge}

Rapid de-escalation of empirical broad-spectrum antimicrobial therapy is an important goal in the prevention of multidrug-resistant bacteria selection. The timely microbiologic diagnosis of ventilator-associated pneumonia could lead to more de-escalation of empirical antimicrobial therapy.

\section{What this paper contributes to our knowledge}

Direct specimen testing onto bronchoalveolar lavage samples at hour 10 was found to be feasible. Early testing (at hour 10) provided valuable information in 18 of the 28 subjects with ventilator-associated pneumonia. This could potentially lead to faster de-escalation of empirical antibiotic therapy in many patients.

Claix, France) according to The European Committee on Antimicrobial Susceptibility Testing recommendations. The second BAL sample $(10 \mathrm{~mL})$ was sent to our laboratory, where it was subjected to centrifugation (4,000g for $4 \mathrm{~min}$ ). Once the supernatant was carefully removed, a $1.5-\mathrm{mL}$ aliquot was used to inoculate 2 Müller-Hinton and 1 blood agar plates. A set of 9 different E-test strips, including amoxicillin, amoxicillin plus clavulanate, cefotaxime, cefepime, piperacillin, piperacillin-tazobactam, ertapenem, doripenem, and colimycin (BioMérieux, Marcy-L'étoile, France), was directly applied to the Müller-Hinton agar plates. Vancomycin and cefoxitin E-test strips were applied onto the blood agar plate (Fig. 1). The Müller-Hinton agar plates were incubated at $37^{\circ} \mathrm{C}$, whereas the blood agar plates were in a $5 \% \mathrm{CO}_{2}$ incubator at $37^{\circ} \mathrm{C}$.

\section{Interpretation of the Results}

Direct Specimen Testing Reading Method. After 10-h and 24-h incubation time, each combination between a single strain and a single agent was assessed. All the results were read blind and offline by 2 independent readers ( $\mathrm{AB}$ and $\mathrm{FM}$ ) to avoid any influence by the Gram stain and/or routine diagnostic laboratory results. Discordant results between the readers were resolved by consensus. Only clinical samples that attained the recommended infective threshold $\left(10^{4} \mathrm{CFU} / \mathrm{mL}\right)$ according to the routine laboratory results ${ }^{9}$ and/or considered as clinically relevant and leading to an antimicrobial therapy decision by ICU clinicians were included a posteriori in the study.

Potential Influence of Direct Specimen Testing on Antibiotic Treatment Options. The E-test approach results were not reported to the ICU clinicians in real time. The 


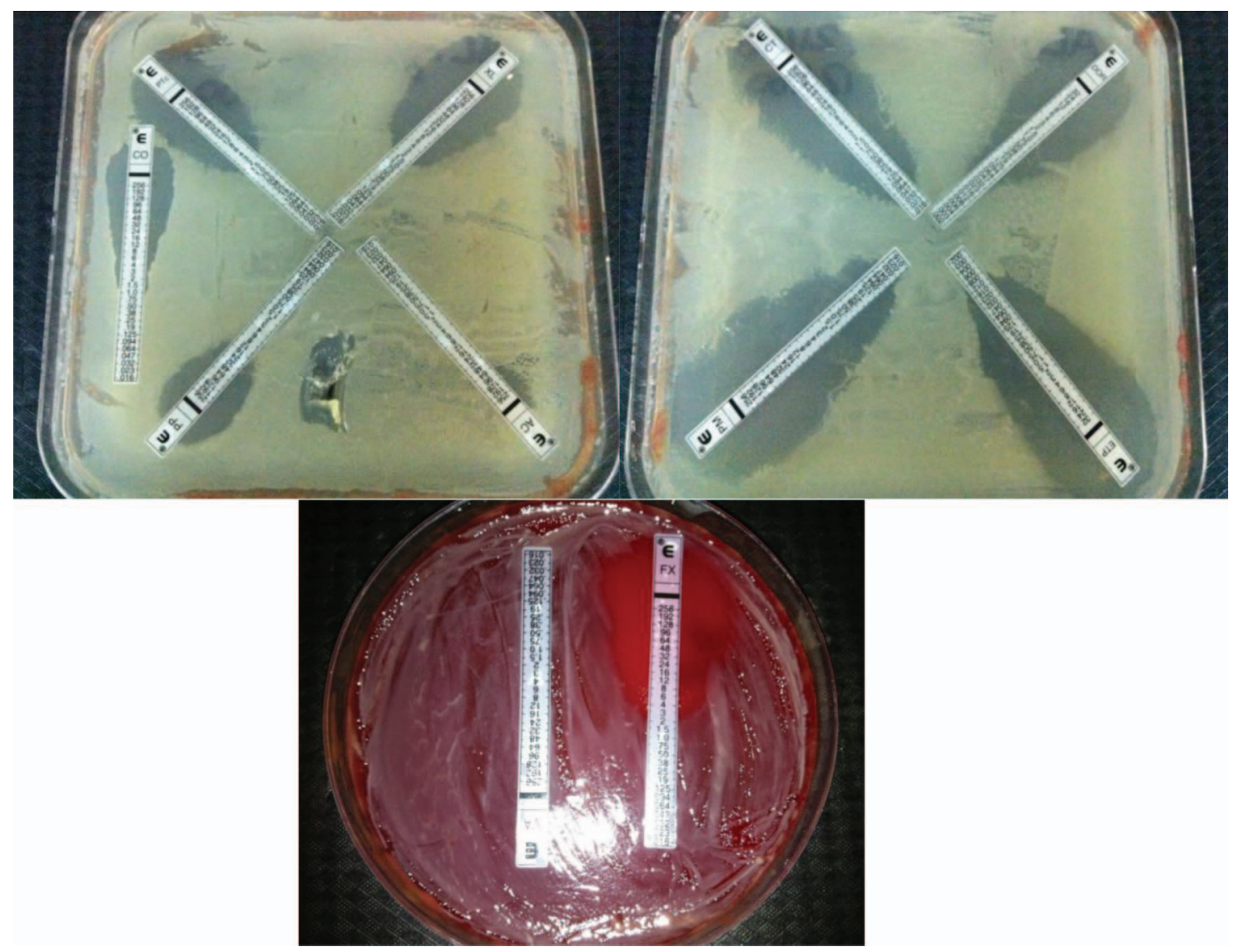

Fig. 1. An example of hour 10 direct specimen testing of bronchoalveolar lavage sample in a subject suspected of having ventilatorassociated pneumonia. E-test strips are placed directly onto agar plates seeded with bronchoalveolar lavage samples.

investigators assessed the E-test results to identify potential drug choices for each case by simulating clinical decision making had the microscopy results been available at the point of care.

Statistical Analysis. Demographic and clinical characteristics of the subjects included in the study and laboratory results were described by using mean \pm SD or median (interquartile range) for quantitative variables and percentages for qualitative variables. The hypothetical drug choices when using the E-test approach were compared for concordance with the narrowest adequate antimicrobial therapy according to the minimum inhibitory concentrations (MICs) provided by the reference method (ie, the routine diagnostic laboratory). The choice of the readers was retrospectively classified as adequate initial antibiotic therapy needing no further modification, adequate initial but broad-spectrum antibiotic therapy that could have benefited from early de-escalation, or inadequate initial antibiotic therapy that needed adjustment. Diagnostic accuracy (sensitivity, specificity) of the E-test method was evaluated as well as its concordance with the reference method by using Cohen's kappa coefficient. A major error of the E-test method was defined when the result indicated intermediate or resistant, whereas the laboratory routine diagnostic method indicated susceptible. A very major error was defined when the E-test method indicated susceptible, whereas the reference method indicated intermediate or agreement between the readers' and bedside physicians' choices (based on the VAP treatment protocol available in the ICU) was then described (the assumptions were not met for performing concordance analyses).

\section{Results}

\section{Population}

Of 57 patients suspected of having VAP, 28 subjects met our VAP criteria and, therefore, were included in this study. The mean $\pm \mathrm{SD}$ age was $54 \pm 19 \mathrm{y}$, the sex ratio was 3.7, and the mean \pm SD SAPS II and SOFA scores were $61 \pm 22$ and $9.5 \pm 3.1$, respectively. The median duration of ventilation at the time of diagnosis was 12 (interquartile range, 5-21) d. At the time of suspicion, the mean \pm SD Clinical Pulmonary Infection Score was $7.0 \pm 1.2$. The reason for admission was impaired con- 


\section{TAILORING EMPIRICAL ANTIMICROBIAL Therapy IN VAP}

sciousness in 11 subjects (39\%), acute respiratory insufficiency in $10(36 \%)$, septic shock in $4(14 \%)$, and other miscellaneous reasons in $3(11 \%)$.

\section{Routine Diagnostic Laboratory Results}

VAP episodes were due to monomicrobial infections in 15 of 28 subjects (54\%). Forty-eight microorganisms recovered from the 28 BAL cultures were considered responsible for VAP infection. Staphylococcus species $(n=9)$ were the most frequently isolated species, followed by Pseudomonas aeruginosa $(n=8)$, Klebsiella pneumoniae $(n=7)$; Enterobacter species, Acinetobacter species, Streptococcus species $(n=4)$; ampicillin-sensitive Enterococcus faecalis $(n=3)$; Haemophilus influenza, Serratia Marcescens, Corynebacterium species $(n=2)$; and other Gram-negative bacteria $(n=3)$. Among Gram-negative bacteria, the prevalence of antibiotic resistance (intermediate or resistant) was $27 \%$ for cefepime; $17 \%$ for piperacillin-tazobactam, ciprofloxacin, and imipenem; and 10\% for amikacin; and all the strains were susceptible to colistin.

\section{Direct Specimen Testing Results at Hour 10}

At hour 10, direct specimen testing was fully or partially readable (not all the combinations were readable, mainly because of the selective growth of some microorganisms, ie, on blood agar plates only) in 18 of the 28 BAL samples (64\%), which corresponded to 33 of 48 strains (69\%). Among monomicrobial VAP $(n=15), 8$ of 15 provided readable BAL cultures ( $n=8$ strains) according to direct specimen testing, whereas 10 of 13 polymicrobial VAP were readable $(n=25$ strains). Among the theoretical 363 microorganism-antimicrobial combinations (11 strips $\times 33$ strains), 90 combinations $(25 \%)$ were not readable for technical reasons (ie, inappropriate disposition of the E-test on the plate, lack of availability of E-test strips, or lack of MIC confirmation by the routine diagnostic laboratory), which provided a total of 273 microorganism-antimicrobial combinations available for reading and analysis.

Overall, the total observed agreement between the hour 10 direct specimen testing approach and laboratory routine diagnostic methods was $90 \%$, with a sensitivity of $83 \%$ and a specificity of $95 \% .8 \%(n=21)$ major error (the E-test method indicated intermediate or resistant, whereas the laboratory routine diagnostic method indicated susceptible), and 3\% $(n=7)$ very major errors (the E-test method indicated $S$, whereas the reference method indicated intermediate or resistant) were observed (Table $1)$. The concordance between the 2 tests was very good $($ kappa $=0.79)$. The overgrowth by which one resistant microorganism prevented the detection of the other(s) was responsible for 20 of 21 major errors (95\%) and occurred only in polymicrobial BAL samples. In addition, 24-h di- rect specimen testing showed $92 \%$ agreement with $10-\mathrm{h}$ direct specimen testing.

\section{Potential Impact of 10-h Reading on Antibiotic Therapy Options}

According to the Results of the Reference Method (ie, the routine diagnostic laboratory). In the 18 subjects for whom BAL samples were readable by E-test, if the 10-h results were to be considered, then $89 \%$ (16/18) of the empirical antibiotics chosen by the blinded readers $(\mathrm{AB}$ and FM) would have been adequate according to the MICs provided by the reference method. At hour 10, the decision of the blinded reader based on the E-test approach results would have provided the following: adequate empirical antibiotic therapy, which would need no further modification in 9 cases; adequate but broad-spectrum empirical antibiotic therapy which would allow de-escalation in 8 cases; and inadequate empirical antibiotic therapy, which would need tailoring in 1 case. In the latter case, at hour 10, the blinded readers reported 2 susceptible bacteria (probably Gram-negative) and a possible Gram-positive microorganism. They thus would have proposed a cefotaxime plus vancomycin therapy. In this particular case, according to the reference method, 3 species were finally considered as responsible for the VAP episode: a wild-type Corynebacterium species $\left(10^{5} \mathrm{CFU} / \mathrm{mL}\right)$, a wildtype Klebsiella species $\left(6 \times 10^{4} \mathrm{CFU} / \mathrm{mL}\right)$, and an Enterobacter species $\left(3 \times 10^{4} \mathrm{CFU} / \mathrm{mL}\right)$. The latter microorganism was resistant to cefotaxime, and the correct choice according to the reference method would have been a carbapenem monotherapy (which was also the choice of the physician in charge of the subject).

In Comparison With the Decision of the Bedside Physician. At the bedside, adequacy of empirical antibiotic therapy according to the MICs of reference was achieved in $94 \%$ subjects $(17 / 18)$ by the ICU physicians. When considering the 10-h E-test approach, the blinded readers ( $\mathrm{AB}$ and FM) would have made the de-escalation as early as $10 \mathrm{~h}$ from receiving the clinical samples in 10 of 18 cases $(55 \%)$ (eg, a subject received piperacillin-tazobactam as the empirical choice of the physician in charge of the patient, and the blinded readers selected cefotaxime at hour 10 after blinded reading of the E-test approach). Although these 10 cases of de-escalations would have been routinely achieved by the physicians in charge of the patient at only hour 72 . In 6 other cases (33\%), the choices made by both the 10 -h blinded readers and the ICU physicians were identical (eg, a subject received cefotaxime as the empirical choice of the physician in charge of the subject and the blinded readers selected cefotaxime at hour 10 after blinded reading of the E-test approach). In the last 2 cases, however, the choice of the 10-h blinded readers was broader than the ICU physicians' choice. In- 


\section{TAILORING EMPIRICAL ANTIMICROBIAL Therapy IN VAP}

Table 1. Cases of Very Major Error Between Hour-10 E-Test Approach and the Diagnostic Laboratory Results

\begin{tabular}{|c|c|c|c|c|c|c|}
\hline \multirow{2}{*}{ Subject No. } & \multirow{2}{*}{ Antibiotic } & \multirow{2}{*}{$\begin{array}{l}\text { Strains Detected } \\
\text { by E-Test }(n)\end{array}$} & \multirow{2}{*}{$\begin{array}{c}\text { Strains Detected } \\
\text { by the Diagnostic } \\
\text { Laboratory (no. and type) }\end{array}$} & \multicolumn{2}{|r|}{ MIC } & \multirow{2}{*}{$\begin{array}{c}\text { Impact on the Adequacy } \\
\text { of the Empirical } \\
\text { Antimicrobial Therapy } \\
\text { Indicated by Hour-10 Reading }\end{array}$} \\
\hline & & & & $\begin{array}{c}\text { ETEST } \\
\text { (BioMérieux) }\end{array}$ & Diagnostic Laboratory & \\
\hline \multirow[t]{4}{*}{6} & Colimycin & 1 & $3^{*}$ & & & None $\dagger$ \\
\hline & & & Pseudomonas aeruginosa & 1.5 & $<0.5$ & \\
\hline & & & Haemophilus influenza & ND & $<0.5$ & \\
\hline & & & Staphylococcus aureus & ND & Resistant & \\
\hline \multirow[t]{4}{*}{8} & Colimycin & 1 & $3 \ddagger$ & & & None§ \\
\hline & & & Enterobacter cloacae & 0.064 & Susceptible & \\
\hline & & & Enterobacter cloacae & ND & Susceptible & \\
\hline & & & Streptococcus species & ND & Resistent & \\
\hline \multirow[t]{5}{*}{$9 \|$} & & 1 & 2 & & & \\
\hline & Cefepime & & Klebsiella pneumoniae & 4 & 4/resistant & YesII \\
\hline & Cefoxitin & & Stenotrophomonas maltophilia & 2 & 8/resistant & \\
\hline & Ertapenem & & & 0.016 & $0.12 /$ resistant & \\
\hline & Doripenem & & & 0.016 & $0.016 /$ resistant & \\
\hline \\
\hline $\begin{array}{l}4 \text { corresponds to } \\
\text { * Three strains } \\
\text { E-test placed on } \\
\text { † The blinded re: } \\
\text { † The same situa } \\
\text { § Piperacillin-taz } \\
\text { very major error } \\
\text { || The slow growt } \\
\text { IT There was a po } \\
\text { ND = not detect } \\
\text { MIC = minimum } \\
\text { MH = Mueller H }\end{array}$ & $\begin{array}{l}\text { the diagnostic lab } \\
\text { ere identified by t } \\
\text { the MH agar plate } \\
\text { aders and the beds } \\
\text { tion occurred for } \\
\text { obactam was used } \\
\text { would not have in } \\
\text { th of a Stenotroph } \\
\text { tential impact bec } \\
\text { ed } \\
\text { a inhibitory concer } \\
\text { finton }\end{array}$ & $\begin{array}{l}\text { oratory MIC of Cefepim } \\
\text { the routine diagnostic lab } \\
\text { e, which corresponded to } \\
\text { ide clinicians had both ch } \\
\text { subject } 8 ; 2 \text { different stra } \\
\text { at the bedside; cefotaxin } \\
\text { npacted the subject's ma } \\
\text { omonas species strain at } \\
\text { ause a carbapenem was t } \\
\text { tration }\end{array}$ & $\begin{array}{l}\text { e for Klebsiella pneumoniae. Resistant corre } \\
\text { oratory: } 2 \text { colimycin-susceptible strains }(P \text {. } \\
\text { the } 2 \text { (overlapping) colimycin-susceptible } P \\
\text { hosen imipenem plus vancomycin: therefore } \\
\text { ins of Enterebacter cloacae and } 1 \text { Streptoce } \\
\text { ne was selected by the blinded readers. On } \\
\text { nagement. } \\
\text { hour } 10 \text { prevented its detection and resulted } \\
\text { the blinded readers' suggestion, whereas a in }\end{array}$ & $\begin{array}{l}\text { sponds to the diagno } \\
\text { leruginosa and } H \text {. in } \\
\text { eeudomonas strains; } \\
\text { this very major erro } \\
\text { ccus species were rec } \\
\text { he basis of the antibi } \\
\text { in very major errors } \\
\text { trinsically carbapene }\end{array}$ & $\begin{array}{l}\text { tic laboratory MIC of Cefepime } \\
\text { luenzae) and S. aureus. Only } 1 \\
\text { aureus was not detectable by t } \\
\text { would not have impacted the st } \\
\text { overed. } \\
\text { ogram, cefotaxime had a good ac } \\
\text { for } 4 \text { antimicrobial-microorganis } \\
\text { n-resistant strain was involved. }\end{array}$ & $\begin{array}{l}\text { for Stenotrophomonas maltophilia. } \\
\text { llipse was observed on the colimycin. } \\
\text { he E-test approach. } \\
\text { abject's management. } \\
\text { tivity against all } 3 \text { strains; therefore, this } \\
\text { m combinations. }\end{array}$ \\
\hline
\end{tabular}

deed, in 1 case, vancomycin was added by the blinded readers because of the suspicion of a resistant Gram-positive bacteria, which finally was identified by the routine diagnostic laboratory as a beta-lactam-susceptible Corynebacterium species. In the other case, the 10-h blinded readers selected piperacillin-tazobactam instead of piperacillin alone (which was the ICU physician's choice).

\section{Discussion}

This study provided 2 clinically relevant results regarding the direct specimen testing approach for BAL samples in the VAP diagnosis. First, it provides relevant microbiologic data at hour 10 in two thirds of the subjects with VAP; and second, these results might lead to early (hour 10) de-escalation of empirical antibiotic therapy in $55 \%$ of the cases compared with the use of standard laboratory techniques (hour 72). The results obtained by direct specimen testing at hour 10 showed $90 \%$ agreement with the diagnostic laboratory results. In addition, direct specimen testing at $24 \mathrm{~h}$ showed $92 \%$ agreement in this study, which was comparable with those obtained in our previous study (89\%) and those reported by several other investigators. $5,7,10,11$
Major errors were also observed in the same proportion as previous studies and were caused by the overgrowth phenomenon. This was not an issue because clinicians will tend to select a drug that targets the less-susceptible microorganism. With regard to very major errors $(2 \%)$, our results at hour 10 were in agreement with those of our previous study $(1.5 \%)$ and with those in the literature (1.03\% in Cercenado et al, ${ }^{5} 1.7 \%$ in Kontopidou et al, ${ }^{10}$ and $3.6 \%$ in Zebouhe et $\left.\mathrm{al}^{11}\right) .{ }^{7}$ With regard to the future routine use of this approach, blood agar plates would be recommended as this rich media and would allow the growth of a maximum of microorganisms in comparison with the Mueller Hinton media. The other reason for generating very major error was the difference in 2 distinct MICs values between hour 10 and the routine laboratory results. Indeed, in this particular case, the MIC values were higher than the break point, ie, resistant in the diagnostic laboratory and below the break point by E-test, although only 2-fold dilutions were observed in both cases.

Because conventional microbiologic reports are rarely provided to clinicians before 48-72 h,6,12 implementing an available and simple approach to clinicians for point-ofcare testing may enable them to evolve from empirical antibiotic treatment to early pathogen-targeted therapy. To 


\section{TAILORING EMPIRICAL ANTIMicrobial Therapy IN VAP}

our knowledge, this was the first study to report data as soon as $10 \mathrm{~h}$ after microbiologic specimen collection. Our study included subjects with late-onset VAP, which led clinicians to the selection of a broad-spectrum antibiotic therapy, whereas the organisms responsible for VAP remained susceptible for the majority of cases. Consequently, we did not encounter any cases in which escalation of antibiotic therapy was required. On the contrary, a relevant percentage of VAP episodes should have been subjected to an early de-escalation strategy.

The exposure time of antibiotics sufficient to induce resistance is unknown, but there should be an advantage to reducing the exposure of the patient to broad empirical antibiotic therapy, which was applicable in 18 of the 28 subjects in our study. ${ }^{9}$ Conversely, in 10 of the 28 subjects with no available reading at hour 10 , no de-escalation was possible but the fact remains that this was neither possible in 24-h nor in the standard groups.

Our study had some limitations. This approach requires a simultaneous BAL and microbiologic procedure to be feasible, therefore, some VAP suspicion during the night shift could be missed. This was a single-center study, and we could not discard a selection bias in favor of a more emblematic VAP diagnosis. Also, direct specimen testing by E-test lacked standardization because the inoculum size was not known and thus could lead to an incorrect MIC value. However, this could actually be viewed as an advantage. Indeed, the E-test method assesses the interaction between microorganisms and antimicrobials in conditions closer to the in vivo situation and, in the case of this study, in pulmonary infections. ${ }^{6-10}$

\section{Conclusions}

This study described a promising method for early direct specimen testing of BAL samples from subjects with VAP. This rapid susceptibility testing approach provided valuable information to clinicians and led to an early adjustment of empirical antimicrobial treatment, which also contributed to a better antimicrobial stewardship. More studies are required to further assess this innovative approach before its potential implementation in real-life patient management.

\section{ACKNOWLEDGMENTS}

We thank the subjects and the clinical and clerical teams involved in this study. We thank Delphine Anquetil for her help in the collection of data.

\section{REFERENCES}

1. Ibrahim EH, Ward S, Sherman G, Schaiff R, Fraser VJ, Kollef MH. Experience with a clinical guideline for the treatment of ventilatorassociated pneumonia. Crit Care Med 2001;29(6):1109-1115.

2. Leone M, Garcin F, Bouvenot J, Boyadjev I, Visintini P, Albanèse J, et al. Ventilator-associated pneumonia: breaking the vicious circle of antibiotic overuse. Crit Care Med 2007;35(2):379-385; quiz 386.

3. Luna CM, Vujacich P, Niederman MS, Vay C, Gherardi C, Matera J, Jolly EC. Impact of BAL data on the therapy and outcome of ventilator-associated pneumonia. Chest 1997;111(3):676-685.

4. Kollef MH. Broad-spectrum antimicrobials and the treatment of serious bacterial infections: getting it right up front. Clin Infect Dis 2008;47(Suppl 1):S3-S13.

5. Cercenado E, Cercenado S, Marín M, Rico MV, Vicente T, Bouza E. Evaluation of direct E-test on lower respiratory tract samples: a rapid and accurate procedure for antimicrobial susceptibility testing. Diagn Microbiol Infect Dis 2007;58(2):211-216.

6. Bouza E, Torres MV, Radice C, Cercenado E, de Diego R, SánchezCarrillo C, Muñoz P. Direct E-test (AB Biodisk) of respiratory samples improves antimicrobial use in ventilator-associated pneumonia. Clin Infect Dis 2007;44(3):382-387.

7. Boyer A, Medrano J, Mzali F, Balick-Weber CC, Bessède E, et al. Direct testing of bronchoalveolar lavages from ventilator-associated pneumonia patients. Diagn Microbiol Infect Dis 2012;73(2):107110.

8. Chastre J, Fagon JY. Ventilator-associated pneumonia. Am J Respir Crit Care Med 2002;165(7):867-903.

9. De Waele JJ, Bassetti M, Martin-Loeches I. Impact of de-escalation on ICU patients' prognosis. Intensive Care Med 2014;40(10):15831585.

10. Kontopidou F, Galani I, Panagea T, Antoniadou A, Souli M, Paramythiotou E, et al. Comparison of direct antimicrobial susceptibility testing methods for rapid analysis of bronchial secretion samples in ventilator-associated pneumonia. Int J Antimicrob Agents 2011;38(2): 130-134.

11. Zebouh M, Thomas C, Honderlick P, Lemee L, Segonds C, Wallet F, Husson MO. Evaluation of a new E-test method for antimicrobial sensitivity testing of Pseudomonas aeruginosa isolates from cystic fibrosis. Pathol Biol (Paris) 2005;53(8-9):490-494.

12. Douglas IS, Price CS, Overdier KH, Wolken RF, Metzger SW, Hance KR, Howson DC. Rapid Automated Microscopy for Microbiological Surveillance of Ventilator-associated Pneumonia. Am J Respir Crit Care Med 2015;191(5):566-573. 